\title{
Taming Web Views in the Detection of Android Privacy Leaks
}

\author{
Xue Qin \\ xue.qin@utsa.edu \\ The University of Texas at San \\ Antonio \\ San Antonio, Texas \\ Xiaoyin Wang \\ xiaoyin.wang@utsa.edu \\ The University of Texas at San \\ Antonio \\ San Antonio, Texas
}

\author{
Robert Neuhaus \\ robert.neuhaus@my.utsa.edu \\ The University of Texas at San \\ Antonio \\ San Antonio, Texas \\ Travis Breaux \\ breaux@cs.cmu.edu \\ Carnegie Mellon University \\ Pittsburgh, Pennsylvania
}

\author{
Diego Gonzales \\ diego.gonzales@my.utsa.edu \\ The University of Texas at San \\ Antonio \\ San Antonio, Texas \\ Jianwei Niu \\ jianwei.niu@utsa.edu \\ The University of Texas at San \\ Antonio \\ San Antonio, Texas
}

\begin{abstract}
Billions of smartphone users force both technical and non-technical facilities to publish their applications in market. One of the easiest ways to create an application for non-techies is by transferring their existing website using WebView. Current approaches for privacy policy analysis are mainly focused on APIs and predefined user interfaces and cannot cover these type of information. In this paper, we proposed a novel solution to trace the input data that is collected from WebView.
\end{abstract}

\section{CCS CONCEPTS}

- Security and privacy $\rightarrow$ software and application security.

\section{KEYWORDS}

android applications, privacy policies, violation detection

\section{ACM Reference Format:}

Xue Qin, Robert Neuhaus, Diego Gonzales, Xiaoyin Wang, Travis Breaux, and Jianwei Niu. 2019. Taming Web Views in the Detection of Android Privacy Leaks. In Hot Topics in the Science of Security Symposium (HotSoS), April 1-3, 2019, Nashville, TN, USA. ACM, New York, NY, USA, 2 pages. https://doi.org/10.1145/3314058.3317732

\section{INTRODUCTION}

Existing work by Slavin et al. [2] attempts to trace privacy policy statements about collection of platform information to API calls with static program analysis. These API calls concern personal data that is automatically collected from the device, such as user location, device identifiers, contact information, and sensor data. Prior work by Wang [3] focuses on tracking privacy policy claims based on direct user input. The user interfaces related to these inputs are generated either statically or programmatically at runtime. However, these works are limited because they did not address personal data that collected through WebView generated user interfaces, which is another major source of private information.

Permission to make digital or hard copies of part or all of this work for personal or classroom use is granted without fee provided that copies are not made or distributed for profit or commercial advantage and that copies bear this notice and the full citation on the first page. Copyrights for third-party components of this work must be honored

For all other uses, contact the owner/author(s).

HotSoS, April 1-3, 2019, Nashville, TN, USA

(c) 2019 Copyright held by the owner/author(s)

ACM ISBN 978-1-4503-7147-6/19/04 ..\$15.00

https://doi.org/10.1145/3314058.3317732
Traditional graphic user interface (GUI) can be implemented using static declarations in resource files, or programmatically in the code. But they are all generated locally. WebView displays web pages as part of the GUIs by loading URLs and it also supports the interaction in the opposite direction, from JavaScript to Java. In other words, the GUIs that is generated remotely by server makes static analysis impossible.

Nowadays, many technical teams provide services for non-technical organizations such as universities and hospitals to take their existing web applications and convert them into mobile apps with the help of WebView. Company like GoNative.io ${ }^{1}$ helped their clients publish $600+$ apps with a $98.1 \%$ approval rating in 2018 . To address the problem, we propose a novel approach to trigger the application loading WebView at runtime while tracing the information flows among the WebView interfaces.

\section{APPROACH}

Our approach contains three major parts. The first part is to develop a tool to automatically explore the Android application while supporting both traditional and WebView GUI analysis. The second part is the procedure of WebView interface detection, we need to identify and modify all the interfaces statically so that they can be recognized by our GUI explorer at runtime and keep track of the information types. The last part is about data flow monitoring, we apply all the potential interface methods to Flowdroid [1] to trace information flow.

\subsection{GUI Exploration}

In order to automatically explore the GUIs of an Android application, we develop a GUI automation tool based on a test automation framework Appium with combination of Android Debug Bridge $(\mathrm{adb})$ and Android UIAutomator. The fundamental goal of our tool is to replace the manual exploration as well as to support WebView GUI analysis.

The tool follows a series of steps to explore: First, our tool takes application as input and preprocess the setups on Appium. For the app that requires login, we provide a manual script that contains login information such as username and password; Then, our tool tries to trigger every possible GUI event on the screen by clicking and entering input data. Subsequently, our tool keeps track of all

$\overline{{ }^{1} \text { https://gonative.io/about }}$ 
the GUI events it has been explored and marks down all the visited WebView within a timeout threshold. The final step is to report the visited record.

\subsection{WebView Interface Detection}

WebView provides a mechanism for the JavaScript code inside it to invoke Android apps' Java code. The API used for this purpose is called addfavascriptInterface(). Android applications can register Java objects to WebView through this API, and all the public methods in these Java objects can be invoked by the JavaScript code from inside WebView. Since Android API 17, all Java methods must include the @favascriptInterface annotation to be accessible within JavaScript.

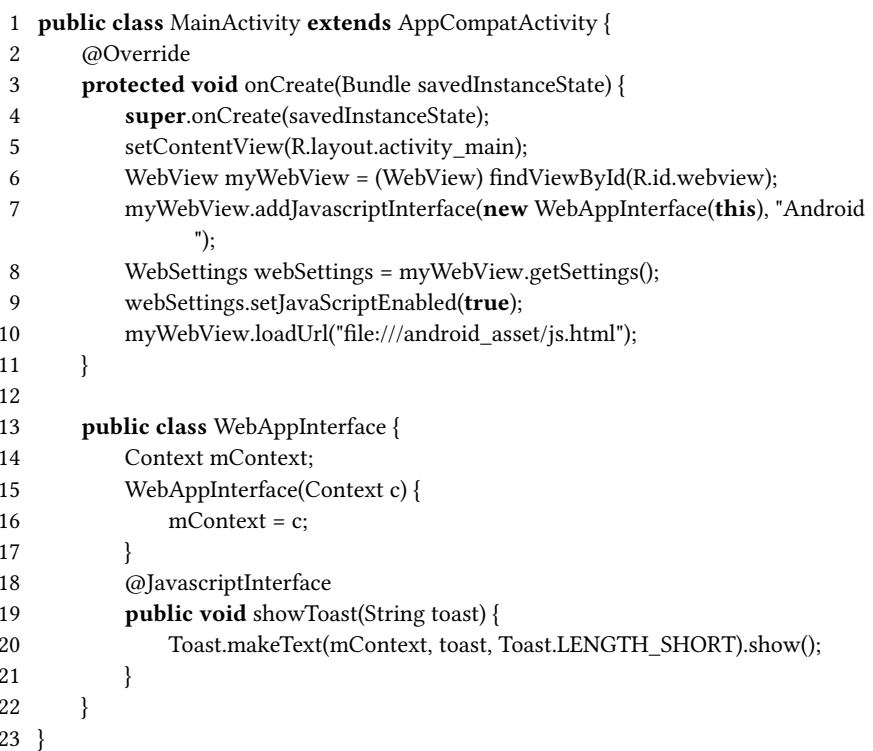

\section{Listing 1: Java Code of WebView Interface Implementation}

Listing 1 shows a GUI implementation example using WebView. keyword@favascriptInterface in line 18 annotates the showToast() method of the WebAppInterface class, and Line 7 illustrates the use of the addfavascriptInterface() API method with an instance of the WebAppInterface class included as a parameter. The addfavascriptInterface() method allows the WebAppInterface instance to be accessible from within JavaScript, while the @favascriptInterface annotation allows JavaScript to call the instance's showToast() method. Line 6 in Listing 2 illustrates the showToast() method of the WebAppInterface instance being called from within JavaScript, passing in a string as a parameter. Without the @favascriptInterface annotation, the showToast() method would not be callable from within JavaScript.

The fact that any method that is called within JavaScript must contain a specific annotation means that a list of methods to monitor can be generated by decompiling an Android application and parsing the resulting .smali files for methods containing the annotation. Figure 1 shows us the frequency of WebView annotation usage from top 100 apps across 8 categories. From the result, $74 \%$ of the apps are using this annotation. And 20 applications each consisted of at least 25 methods containing the annotation, and 5 applications have more than 100 annotations.

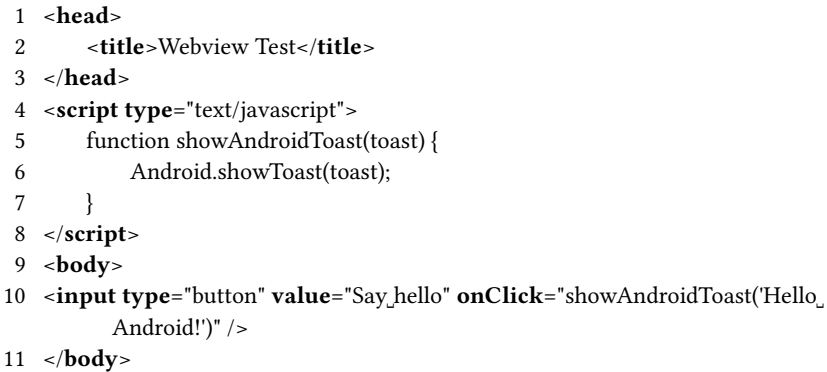

Listing 2: Sample JavaScript Code of a Java Object's Method Being Called

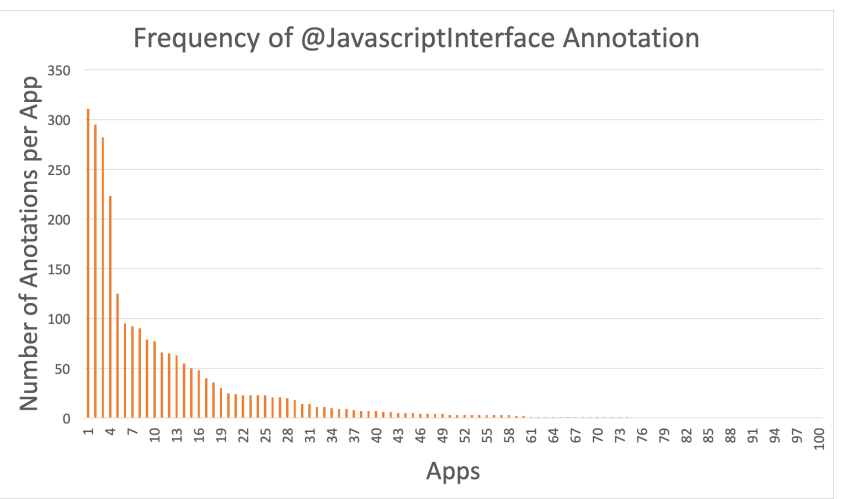

Figure 1: Frequency of @JavascriptInterface Annotation

\subsection{Data Flow Monitoring}

After we identify the all web view interface method invocations between JavaScript code and Android Java code, we will instrument the invocations and record the data transferred through the interface. During the GUI exploration, we input a unique value into each different text box, so that we can tell the source of the data based on the value. Therefore, for each interface invocation, we can tell which text boxes are its data source. Finally, applying FlowDroid to the Android Java code with the interface invocations as information sources, we can tell input of which text boxes in the web views flows to the network.

\section{REFERENCES}

[1] Steven Arzt, Siegfried Rasthofer, Christian Fritz, Eric Bodden, Alexandre Bartel, Jacques Klein, Yves Le Traon, Damien Octeau, and Patrick McDaniel. 2014. FlowDroid: Precise Context, Flow, Field, Object-sensitive and Lifecycle-aware Taint Analysis for Android Apps. SIGPLAN Not. 49, 6 (Jun 2014), 259-269. https://doi.org/10.1145/2666356.2594299

[2] Rocky Slavin, Xiaoyin Wang, Mitra Bokaei Hosseini, James Hester, Ram Krishnan, Jaspreet Bhatia, Travis D. Breaux, and Jianwei Niu. 2016. Toward a Framework for Detecting Privacy Policy Violations in Android Application Code. In Proceedings of the 38th International Conference on Software Engineering (ICSE '16). ACM, New York, NY, USA, 25-36. https://doi.org/10.1145/2884781.2884855

[3] X. Wang, X. Qin, M. Bokaei Hosseini, R. Slavin, T. D. Breaux, and J. Niu. 2018. GUILeak: Tracing Privacy Policy Claims on User Input Data for Android Applications. In 2018 IEEE/ACM 40th International Conference on Software Engineering (ICSE). 37-47. 